\title{
Influence of Sensory Cues on the Stimulability for Liquid Sounds in Brazilian Portuguese-Speaking Children
}

\author{
Márcia Mathias de Castro ${ }^{a}$ Haydée Fiszbein Wertzner ${ }^{b}$ \\ a University of São Paulo School of Medicine, b Speech-Language Pathology and Occupational Therapy \\ Department, University of São Paulo School of Medicine, São Paulo, Brazil
}

Key Words

Speech · Speech articulation tests - Speech disorders

\begin{abstract}
Objective: Stimulability is the ability to produce an adequate sound under specific conditions. This study aimed to describe the stimulability of Brazilian Portuguese-speaking children with and without phonological disorders for the production of liquid sounds with the aid of visual and tactile cues. Patients and Methods: The study sample included 36 children between 5;0 and 11;6 years of age, 18 with phonological disorder and 18 without any speech-language disorders. Stimulability was measured for syllable imitation. The stimulability test employed includes 63 syllables with the sounds $[1],[K]$, and $[r]$, as well as seven oral vowels. If the subject was unable to imitate a sound, a visual cue was given. When necessary, a tactile cue was also given. Results: The sound $[K]$ required greater use of sensory cues. Children with phonological disorder needed a greater number of cues. Conclusion: The use of sensory cues seemed to facilitate sound stimulability, making it possible for the children with phonological disorder to accurately produce the sounds modeled.

Copyright $\odot 2009$ S. Karger AG, Basel
\end{abstract}

\section{Introduction}

Children with phonological disorder (PD) are heterogeneous in terms of severity, type of errors, causal factors, and maintenance factors. This heterogeneity has motivated researchers and clinicians to identify deficits in speech processing [1], as well as cross-linguistic interference.

The production of liquid sounds requires articulatory subtlety and sensory motor skills [2]. In Brazilian Portuguese (BP), there are three common liquid sounds $/ 1, \Lambda, f /$ that occur in consonant-vowel, consonant-consonantvowel, and consonant-vowel-consonant syllables [3]. These aspects are reflected during the developmental period, when the liquid simplification process, which is later eliminated, occurs, indicating a demand for maturity, either cognitive or motor [4].

In the literature, the classification of PD is based on correlated causal factors [5] or on linguistic characteristics [1], as well as on the possibility of recovering of difficulties through markers, diagnostic tools used for classification. Individuals with PD can present cognitive difficulties or delayed development of speech organs. In children who present poor motor control, some speech errors represent approximation strategies that can manifest as sound distortion, substitution, or omission, demonstrating unfamiliarity with or inability to produce the target sound $[3,6]$.

The maturational process of articulators occurs sequentially from the lips to the pharynx, the neural net-

Márcia Mathias de Castro

Rua Ana de Proença, 49, Tatuapé

São Paulo, SP 03409-040 (Brazil)

Tel. +55 112942 9134, Fax +55 1130917714

E-Mail mamcastro@usp.br 
works being more complex in the lips and tongue [6]. The ability to produce liquid sounds involves constrictions/ vibrations of the lips and tongue that require maturation of the nervous system.

In children, motor and sensory processes mediate the language environment and feedback. During phonological development, simultaneous and complementary differentiation processes (isolation of characteristics) and integration processes (combining of isolated properties) occur in functional relations [7].

The dynamic system theory explains motor development in terms of the regulation of movement and the learning of motor skills in a narrow and continuous connection between action and perception [2]. The pattern of muscle selection is guided by cues. More specifically, when learning to speak, children learn sequences of movements, together with the sensory consequences of those movements. The sensory information collected can be used to modify sequences or learn new sequences containing some of the same elements of movement. Intrinsic and extrinsic variables combine to impair or facilitate motor responses. During speech, complex sequences of contention and facilitation occur in the anatomical and physiological development of the organs involved. These alterations include severity effects, as well as changes in the mass and geometry of the vocal tract. Therefore, the child produces spontaneous gestures that are learned and refined, eventually being performed with accuracy and efficiency [2].

Sensory information is the basis of the continuous selection and refinement of motor patterns for the production of sounds. The integration of sensory and motor characteristics produces stability at each repetition. The sounds produced are represented in maps of neuronal activity associated with motor activities that are related to the receptors (cutaneous, kinesthetic, auditory, and visual). The phoneme is a global map, a dynamic structure in which connected maps (areas of detection, intention, and movement continuity) interact [7].

Stimulability is the ability to immediately correct errors in speech production when a model is provided through sensory cues. It is the difference between spontaneous sound production and that optimized by imitation. If performance is better in imitation, the subject is considered stimulable for the target sound. The ability to imitate reflects phonological knowledge of the phoneme, implying ability to distinguish between two phonemes based on their linguistic and phonotactic characteristics [8]. Clinicians can use stimulability as a resource to identify which sounds are absent from the repertoire of a child with PD. We know that stimulability can be influenced by maturational, sensory, motor, and visibility factors, as well as by subsequent vowels. Considering the close relationship between perception and production, the sequence of movements has sensory consequences that are processed in order to modify these movements. A child might present age-appropriate production of consonants and vowels without possessing the skills needed to produce those sounds in all configurations required by the language. Final consonants, consonant clusters, and multisyllabic words can be problematic [9]. The stimulability test provides an objective measure that has prognostic indications in the selection of the target sound for intervention in $\mathrm{PD}$. Some authors state that treating a nonstimulable sound favors the introduction of a new element into the phonological knowledge that will reorganize the phonological system [10]. Stimulability is typically defined as the ability of a child to reproduce at least $10 \%$ of the stimuli provided $[8,11]$.

It is recommended that sensory cues (acoustic, visual, and tactile) be used in the evaluation of speech stimulability $[12,13]$, since such cues allow interaction between perception and production of the target sound. Sensory cues imply sensory input, selective attention, and attribution of meaning to a stimulus, as well as organization of response and completion of the performance [2]. Since PD occurs predominantly in children from 5 to 8 years of age (during the maturation process) [4], stimulability evaluation using sensory cues can enable the child to modify existing movement sequences or learn new sequences.

The two hypotheses of this study were that children with PD would not be stimulable for liquid sounds, and that visual sensory cues would be employed more often than would tactile sensory cues for the production of liquid sounds. Our objective was to use sensory cues to describe the stimulability for liquid sounds of BP-speaking children with and without PD.

\section{Method}

The study design was approved by the Ethics Committee of the University of São Paulo School of Medicine Hospital das Clínicas (protocol No. 150/03). Parents or legal guardians gave written informed consent.

A total of 36 children between 5;0 and 11;6 years of age took part in the study: 18 with PD, designated the PD group (PDG), and 18 without any speech-language disorders, constituting a control group (CG). Children in the CG were age- and gendermatched to those in the PDG. The criteria for inclusion in the PDG were presenting altered liquid sounds in consonant-vowel 
syllables and still being diagnosed (or having had less than three therapy sessions). The inclusion criterion for the CG was presenting no language, speech, or hearing problems, as stated on the questionnaire completed by the parents and confirmed through audiological evaluation.

The stimulability test comprises syllable imitation and the possibility of providing sensory cues. The test is composed of 63 syllables, including the lateral phonemes $/ 1$ and $K /$, as well as the simple vibrant phoneme $/ \mathrm{f} /$, combined with the seven oral vowels in three occurrences [3]. Each syllable was produced once by the examiner facing the child in order to allow visualization of the articulation point and the repetition of each syllable. If the child produced the first syllable incorrectly, the visual cue was given only for this syllable; if the production remained incorrect, the tactile cue was given. For the visual cue, the examiner showed the tongue position used in producing each sound and offered the child a mirror to monitor the articulatory movements. If the production was incorrect, the tactile cue was used with a spatula in the place where the tongue touches the palate to produce the sound [11]. These procedures were followed in both groups. A classification system was developed to analyze stimulability. Production of each sound was scored from 0 to 2, 0 indicating nonstimulability, 1 indicating stimulability with sound distortion, and 2 indicating stimulability with correct production. Based on those criteria, the stimulability index was calculated for each sound. The score obtained was divided by the expected total (42) and multiplied by 100 . In cases where sensory cues were necessary, the score was given according to the production after their use. Children reproducing $10 \%$ of the stimuli provided were considered stimulable.

In a different analysis, we noted when a child emitted the target sound correctly at least once during the stimulability test and production improved. We also noted when a child added a vowel before the target sound, modifying the syllable from consonant-vowel to vowel-consonant-vowel, which was considered modification regardless of whether the target sound was produced correctly.

\section{Results}

Most PDG children were stimulable for any of the three sounds: $89 \%$ for $[1], 36 \%$ for $[\Lambda]$, and $33 \%$ for $[\tau]$. The percentage of PDG children requiring the visual cue/tactile cue combination was, respectively, $27 / 22 \%$ for [1], $55 / 44 \%$ for $[\Gamma]$, and $85 / 62 \%$ for $[\Lambda]$. A small percentage of children required the tactile cue, which was most often used for the sound $[K]$. However, the test for equality of proportions, when applied to the use of the sensory cues, revealed no significant differences between the two types of stimuli ( $\mathrm{p}=0.701$ for $[1] ; \mathrm{p}=0.505$ for $[\mathrm{r}]$, and $\mathrm{p}=0.137$ for $[\Lambda])$.

None of the CG children required cues for [1], although 1 child required the tactile cue for $[r]$. The sound that required the greatest use of sensory cues was $[K]: 39 \%$ needed the visual cue, although none needed the tactile cue. The comparison between the two types of sensory cues,
Table 1. Improvements and modifications in sound production by group

\begin{tabular}{|c|c|c|c|c|c|c|}
\hline & \multicolumn{3}{|c|}{ Improved } & \multicolumn{3}{|c|}{ Modified } \\
\hline & {$[1]$} & {$[\boldsymbol{f}]$} & {$[\Lambda]$} & {$[1]$} & {$[\boldsymbol{f}]$} & {$[\kappa]$} \\
\hline \multicolumn{7}{|l|}{$P D G$, yes } \\
\hline Children & 3 & 8 & 10 & 5 & 18 & 17 \\
\hline$\%$ & 16.7 & 44.4 & 55.6 & 27.8 & 100.0 & 94.4 \\
\hline \multicolumn{7}{|l|}{$\overline{P D G}$, no } \\
\hline Children & 15 & 10 & 8 & 13 & 0 & 1 \\
\hline$\%$ & 83.3 & 55.6 & 44.4 & 72.2 & 0.0 & 5.6 \\
\hline $\mathrm{p}$ value & $<0.001^{*}$ & 0.505 & 0.505 & $<0.001^{*}$ & $<0.001^{*}$ & $<0.001^{*}$ \\
\hline \multicolumn{7}{|l|}{$C G$, yes } \\
\hline Children & 0 & 2 & 7 & 1 & 18 & 15 \\
\hline$\%$ & 0.0 & 11.1 & 38.9 & 5.6 & 100.0 & 83.3 \\
\hline \multicolumn{7}{|l|}{$\overline{C G, \text { no }}$} \\
\hline Children & 18 & 16 & 11 & 17 & 0 & 3 \\
\hline$\%$ & 100.0 & 88.9 & 61.1 & 94.4 & 0.0 & 16.7 \\
\hline $\mathrm{p}$ value & $<0.001^{*}$ & $<0.001^{*}$ & 0.182 & $<0.001^{*}$ & $<0.001^{*}$ & $<0.001^{*}$ \\
\hline
\end{tabular}

using the test for equality of proportions, showed $\mathrm{p}=$ 0.546 for $[\mathrm{r}]$ and $\mathrm{p}=0.003$ for $[K]$, demonstrating a significant difference between visual and tactile cue use for $[\Lambda]$. No cues were used for [1], and therefore no comparisons were made.

The majority of the PDG children showed improvement in their production of $[K]$. Improvement indices demonstrated that CG children already presented correct production. Table 1 shows that target sound production was modified primarily for $[\delta]$ and $[\Lambda]$. The most common modification was the addition of a vowel preceding the target sound, as when / ra/ was produced as [ara]. This phenomenon occurred in both groups. Not all children preceded the consonant with the vowel that followed, although some always preceded it with the same vowel, as in [era], [ero] and [eru].

We used a test for equality of proportions to compare the two groups in terms of improvement and modification (table 1). In the PDG, there was no improvement for [1], since it was already produced correctly. For the other sounds, there was no significant difference in terms of improvement. In terms of modification, however, there were significant differences among the three sounds, few PDG children modifying [1], whereas most modified [ $\mathrm{c}]$ and $[\Lambda]$. In the CG, the significant difference for 'no improvement in the production of [1] or [c]' indicates correct production. There was no difference for $[\Lambda]$. In terms of 
modification of sound production, we observed a significant difference among the three sounds, most CG children modifying $[r]$ and $[K]$.

\section{Discussion}

In $\mathrm{BP}$, the three liquid sounds occur frequently. During phonological development, $[r]$ and $[K]$ are acquired between $4 ; 0$ and $4 ; 6$ years of age, whereas [1] is typically acquired at 3;0 years of age [3]. This can explain the fact that our first hypothesis was not confirmed, since most of the PDG children were stimulable for the three liquid sounds of BP: $89 \%$ for $[1] ; 36 \%$ for $[K]$, and $33 \%$ for $[r]$.

Our second hypothesis was partially confirmed. In the PDG, the visual cue was more helpful than the tactile cue for all three sounds. The same was true in the CG, except in relation to [1], for which no sensory cues were required, although the only significant difference was for $[K]$. Of the three liquid sounds, [1] required the fewest visual cues, since only a few children presented difficulty in producing this sound. The sounds $[r]$ and $[K]$ required the use of the auxiliary cues, $[K]$ requiring the greatest number of visual cues.

Other authors have recommended the use of sensory cues as facilitators of sound production [11, 12, 14], demonstrating that children with PD present stimulability for sounds after direct training with sensory cues for a specific sound in imitation tasks [11]. The possibility of providing sensory cues allows clinicians to individualize treatment by identifying the best means of facilitating sound production [14].

The frequent use of visual cues in the PDG is noteworthy and promoted correct production of sounds that presented difficulty for those children, especially of the most visible sounds [1] and [ $[$ ], as the visual cue involves the use of a mirror, encouraging children to monitor their movements [13]. Oral motor control involves a synergy between lips and mandible movements that involve constriction of the vocal tract for the production of the target sound. Therefore, the combination of visual information and acoustic stimuli facilitates adjustment of the articulatory gesture [2].

The dynamic systems theory emphasizes the connection between action (oral motor gesture) and the self-perception that this action creates. Therefore, children develop the ability to monitor the auditory and kinesthetic aspects of articulatory gestures, allowing them to learn the corresponding motor skills through certain pathways.
It is well known that PD occurs during maturation, which must be considered during the evaluation of any child [2]. In the present study, stimulability was greater among older children in both groups. When clinicians offer sensory cues for the execution of the movement, the child begins to present a different organization at each attempt. The synchrony between perception and production allows adjustments that facilitate sound production, even of liquid sounds that require complex motor adjustments. The motor and sensory processes mediate the feedback. Therefore, articulation can be influenced by sensory stimuli, and stimulability can facilitate the use of sensory information to reorganize production through motor gesture adjustments.

In the present study, sensory information appeared to make it possible for children with PD to overcome their difficulties and improve their sound production; even if they did not reproduce the sound correctly immediately after the stimulus, they did so at some point during the task. This indicates that repetition of the target stimulus facilitates the production of a newly introduced sound [15]. Repetition of the acoustic stimulus also plays a role in improving sound production $[12,13]$. The sensory cues might act as extrinsic variables to facilitate the motor responses. We observed that some children in each group, regardless of the use of sensory cues, modified their production by inserting a vowel before $[K]$ or $[r]$. This demonstrates that Brazilian children, with or without PD, have internalized the rule that, in $\mathrm{BP}$, these sounds do not occur in the initial position [3].

In addition to issues related to perception and production, language interferences, such as syllable type and word length, can affect the production of liquid sounds [9]. Clinicians should exercise caution when selecting words containing the target sounds. In minimal pairs, it is possible to oppose stimulable and nonstimulable sounds, thereby shortening the intervention time. It would be interesting to reapply this study in order to identify possible motor difficulties for such production, which can occur in some cases of PD [6].

In conclusion, children with PD were stimulable for all three sounds evaluated and demonstrated knowledge of the phonotactic distribution of these sounds in the language. Sensory cues were required, especially for the sounds $[\Gamma]$ and $[K]$, and seemed to be effective production facilitators. Another production facilitator was the repeated imitation of sounds. The gestures that children produce and learn can be refined through the use of sensory cues, leading to the desired production. 


\section{References}

1 Crosbie S, Holm A, Dodd B: Intervention for children with severe speech disorder: a comparison of two approaches. Int J Lang Commun Disord 2005;40:467-491.

2 Kent RD: Motor control: neurophysiology and functional development; in Caruso A, Strand E: Clinical Management of Motor Speech Disorders in Children. Thieme, New York, 1999, pp 29-70.

3 Castro MM, Wertzner HF: Estimulabilidade e tipos de erros de fala. Rev Soc Fonoaudiol 2006;11:1-9.

4 Wertzner HF: O distúrbio fonológico em crianças falantes do português: descrição e medidas de severidade; Tese Livre Docente Faculdade de Medicina da Universidade de São Paulo, São Paulo, 2002.

5 Shriberg LD: Diagnostic markers for child speech-sound disorders: introductory comments. Clin Linguist Phon 2003;17:501-505.
6 Green JR, Moore CA, Reilly KJ: The sequential development of jaw and lip control for speech. J Speech Lang Hear Res 2002;45:6679.

7 Kent RD: The biology of phonological development; in Ferguson CA, Menn L, StoelGammon C: Phonological Development: Models, Research, Implications. Timonium, York Press, 1992.

8 Powell TW, Miccio AW: Stimulability: a useful clinical tool. J Commun Disord 1996;29: 237-253.

9 Velleman SL: Phonotactic therapy. Semin Speech Lang 2002;23:43-55.

10 Rvachew S: Stimulability and treatment success. Top Lang Disord 2005;25:207-219.
11 Rvachew S, Rafaat S, Martin M: Stimulability, speech perception skills, and the treatment of phonological disorders. Am J Speech Lang Pathol 1999;8:33-43.

12 Hodson BW, Scherz JA, Strattman KH: Evaluating communicative abilities of a highly unintelligible preschooler. Am J Speech Lang Pathol 2002;11:236-242.

13 Khan LM: The sixth view: assessing preschooler's articulation and phonology from the trenches. Am J Speech Lang Pathol 2002; 11:250-254

14 Peña-Brooks A, Hedge MN: Assessment and Treatment of Articulation and Phonological Disorders in Children. Austin, Pro-ed, 2000.

15 Goldstein B, Fabiano L, Iglesias A: Spontaneous and imitated productions in Spanishspeaking children with phonological disorders. Lang Speech Hear Serv Sch 2004;35: 5-15. 